\title{
EDITORIAL
}

\section{Numerical Analysis for Functional Differential and Integral Equations*}

\author{
Hermann BRUNNER ${ }^{1,2}$, Tao TANG ${ }^{2}$, Stefan VANDEWALLE ${ }^{3}$ \\ 1 Department of Mathematics and Statistics, Memorial University of Newfoundland, \\ St. John's, NL A1C 5S7, Canada \\ 2 Department of Mathematics, Hong Kong Baptist University, Hong Kong, China \\ 3 Departement Computerwetenschappen, Katholieke Universiteit Leuven, Celestijnenlaan \\ 200A, B-3001 Leuven-Heverlee, Belgium
}

(C) Higher Education Press and Springer-Verlag 2009

From December 3-6, 2007, the Department of Mathematics at Hong Kong Baptist University hosted the International Workshop on Numerical Analysis and Computational Methods for Functional Differential and Integral Equations. This workshop, organized by Hermann Brunner of Memorial University of Newfoundland (Canada) \& Hong Kong Baptist University, Leevan Ling and Tao Tang of Hong Kong Baptist University, and Chengjian Zhang of Huazhong University of Science and Technology (China) brought together some 40 members of research groups in Hong Kong, Taiwan and the mainland of China, Belgium, Canada, Japan, and Portugal. The aims of the workshop were twofold. First, it is to discuss recent and current work in this active area of numerical analysis/scientific computing. Second, it is to foster closer research contacts and research collaboration between participants.

The eleven papers in this special issue of Frontiers of Mathematics in China reflect the broad spectrum of research work presented during the fourday workshop. Three of the papers are survey papers, dealing with theory and numerical analysis of nonlinear stiff Volterra functional differential equations (Li); collocation methods for pantograph-type delay differential and integral equations (Ishiwata and Muroya, note that Section 4 of the paper also contains some new research results); and current work and open problems in the numerical analysis of Volterra functional equations (Brunner).

The study of various aspects of numerical stability for (continuous and discrete) delay problems is the focus of four papers: they are by Huang and Vandewalle (stability of Runge-Kutta-Pouset methods for Volterra integro-

* Received November 18, 2008

E-mail: brunner@math.hkbu.edu.hk, hermann@math.mun.ca; ttang@math.hkbu.edu.hk, stefan.vandewalle@cs.kuleuven.be 
differential equations with constant delays); by Wang and Li (convergence of Runge-Kutta methods for neutral Volterra integro-differential equations with constant delay); by Tian, Zhang and Sun (delay-independent stability of Euler's method for nonlinear DDEs with constant delay); and by Muroya, Ishiwata and Guglielmi (global stability conditions for a class of difference equations).

Two papers analyze the convergence properties of spectral methods for Volterra integral equations (Wan, Chen and Huang) and for pantograph-type DDEs and Volterra integral equations with multiple delays (Ali, Brunner and Tang).

Finally, the papers by Koto and by Teodoro, Lima, Ford and Lumb deal, respectively, with IMEX linear multistep methods for ordinary and delay differential equations, and with the numerical solution of forward-backward differential equations.

All papers in this special issue were refereed. We would like to thank all referees for their helpful assistance.

Detailed information on the workshop, including the scientific committee, the invited speakers, the organizing committee and the conference program, can be found in

$$
\text { http: //www . math.hkbu . edu.hk/budde }
$$

We gratefully acknowledge the generous financial support provided by Hong Kong Baptist University, K. C. Wong Education Foundation, Lee Hysan Foundation, and Joint Research Institute for Applied Mathematics between Peking University and Hong Kong Baptist University.

\section{Hermann BRUNNER}

Memorial University of Newfoundland, Canada

Hong Kong Baptist University, China

Tao TANG

Hong Kong Baptist University, China

Stefan VANDEWALLE

Katholieke Universiteit Leuven, Belgium 